\title{
Estrategia de búsqueda de información científica aplicada al reintegro laboral de personas con discapacidad
}

\author{
Search strategy for scientific information applied to the return to work of people with disabilities \\ Ana Milena Galarza-Iglesias ${ }^{1 *}$ orcid.org/0000-0001-5643-3700
}

1 Escuela de rehabilitación Humana, Facultad de Salud, Universidad del Valle. Cali, Colombia

\section{Resumen}

Introducción: El ejercicio de la práctica profesional relacionado con el reintegro laboral de personas con discapacidad, debe soportar su actuar en la evidencia científica. Con el avance de las tecnologías de información y comunicación se tiene acceso a un gran acervo de resultados de investigaciones, a las cuales se pueden acceder a través del uso de estrategias de búsqueda. Objetivo: Evidenciar la pertinencia de una estrategia de búsqueda para recuperar publicaciones científicas relacionadas con el reintegro laboral de personas con discapacidad. Materiales y métodos: Se presenta de forma sistemática la estrategia de búsqueda a partir de definición de la pregunta, identificación de términos de búsqueda en lenguaje común y controlado, ecuación de búsqueda, búsqueda-recuperación de artículos, criterios inclusión-exclusión, selección de artículos, lectura crítica y respuesta a pregunta de investigación. Lo anterior puede ser reproducido ajustando descriptores según el interés profesional. Resultados: Se recuperaron 15 artículos que responden a la pregunta ejemplo, evidenciando la pertinencia de la estrategia de búsqueda. Conclusiones: La estrategia de búsqueda de información académica propuesta, permite recuperar artículos que son pertinentes para el investigador y profesionales en su práctica diaria.

Palabras clave: Reinserción al trabajo; persona con discapacidad; trabajo; estrategia de búsqueda; booleano; terapia ocupacional. (Fuente: DeCS, Bireme).

\begin{abstract}
Introduction: The professionals to exercise their practice in an updated and effective way in the matter of return to work of persons with disabilities must support their actions in the scientific evidence. With technology you have access to a lot of information. To be effective and retrieve the data that is required the use of search strategies. Objective: To demonstrate the relevance of a search strategy to recover scientific publications, in this case, labor reimbursement of persons with disabilities. Materials and methods: The search strategy is systematically presented based on the definition of the question, identification of search terms in common and controlled language, search equation, search-retrieval of articles, inclusion-exclusion criteria, selection of articles, critical reading and answer to research question. The above can be reproduced by adjusting descriptors according to professional interest. Results: 15 articles were retrieved that answer the example question evidencing the relevance of the search strategy. Conclusions: An academic information search strategy allows retrieving articles that are relevant for the researcher and professionals in their daily practice.
\end{abstract}

Key words: Return to work; person with disabilities; work; search strategy; Boolean; occupational therapy. (Source: DeCS, Bireme).

*Autor de correspondencia

Ana Milena Galarza Iglesias

e-mail: ana.galarza@correounivalle.edu.co 


\section{Introducción}

A nivel mundial se estima que el $15 \%$ de las personas presenta alguna discapacidad. Esta población tiene menor participación en actividades de la vida diaria que involucran su bienestar integral, como son las relacionadas con la salud, educación, recreación y trabajo(1).

Cuando la discapacidad se presenta en personas que están en el ambiente laboral y luego de un periodo de ausencia, se propone un plan de rehabilitación que tiene como meta el reintegro laboral en alguna de sus modalidades( ${ }^{(2)}$, se genera una serie de acciones donde participan actores de la empresa, así como profesionales de áreas como la medicina, terapia ocupacional, psicología, derecho, administración de negocios, entre otros, lo cual podría generar falta de consenso sobre cómo abordar el reintegro laboral, con qué población o las consideraciones que se debe tener, en especial en el caso de la discapacidad de origen laboral(3).

Es así como al momento de planear el reintegro laboral surgen diferencias sobre cómo debe ser abordado en personas con discapacidad y, para solucionar los interrogantes se propone recurrir a la evidencia científica, de tal manera que se tenga insumos que permitan orientar el proceso de reintegro de la mejor manera.

Una alternativa para los profesionales es sustentar su actuar en la evidencia científica. En la actualidad, con los avances tecnológicos, se tiene acceso a gran cantidad de información, en un primer momento con el uso de motores de búsqueda como GOOGLE ${ }^{(4)}$, sin embargo, no toda la información ha pasado por filtros rigurosos ni por pares académicos. Otra alternativa de búsqueda es a través de Google académico donde se puede recuperar información no sólo de artículos, sino también de tesis, libros, blogs, entre otros (5). Esta situación puede afectar los resultados de la búsqueda en el ámbito investigativo o de práctica profesional, dado que se puede encontrar información desactualizada, sin rigor metodológico, artículos sin contenido relevante que, además de tomar tiempo en su revisión, pueden llevar a conclusiones erróneas. Una búsqueda más específica se puede realizar en Medline a través de Pubmed(6) identificando la temática, sin embargo, al no aplicar una estrategia de búsqueda se asume el riesgo de que al realizar la traducción a otro idioma se pierda el sentido de relación con la temática de la investigación. Para ser efectivos y recuperar los datos que se requieren, es necesario el uso de alguna estrategia de búsqueda de documentos (7).

Para realizar una búsqueda efectiva, es importante establecer una pregunta o tema de investigación(8), una estrategia, términos tanto naturales como normalizados que permitan identificar, acceder y recuperar eficientemente dicha evidencia. Los términos naturales o lenguaje general ${ }^{(9)}$ crean la relación entre el significado, el contexto y una jerarquía, de tal manera que un mismo término puede ser usado de diferentes formas según su contexto. De este lenguaje natural surge el lenguaje especializado, reconocido entre grupo de personas según las áreas de interés y que posee las mismas características, sin embargo, la finalidad del lenguaje es comunicar y por ello, se considera que los dos son necesarios para recuperar el máximo de documentación.

Al hacer búsqueda especializada se requiere de tesauros para tener un lenguaje controlado, organizado y apropiado a la terminología usada en el campo específico del tema. Los tesauros están conformados por descriptores y son usados para la indización de documentos científicos, académicos. Esto permite que la información recuperada sea pertinente(10), y a partir de ellos, con el uso de conectores booleanos, construir una ecuación de búsqueda que puede ser actualizada tanto en términos como en filtros. Posteriormente, se tiene la clave para encontrar con mayor posibilidad la información que aporta a resolver la pregunta planteada.

Es así como la búsqueda y recuperación de información, en este caso de artículos científicos, requiere una estrategia(11). Si bien, se puede desarrollar la práctica profesional a partir de la experiencia y de documentación general, la información científica publicada en artículos ofrece alternativas que se avalan en un medio académico. El objetivo de este estudio es evidenciar la pertinencia de una estrategia búsqueda para recuperar publicaciones científicas, en el tema de reintegro laboral de personas con discapacidad de origen laboral. Al seguir los pasos propuestos en la estrategia se podrá usar los términos que sean de interés al investigador. 


\section{Materiales y métodos}

La metodología utilizada para desarrollar la estrategia de búsqueda siguió un proceso sistemático con los siguientes pasos:

1. Definición de pregunta a resolver.

2. Identificación de los términos de búsqueda: identificación de sinónimos primero en lenguaje común, luego en el diccionario de la Real Academia Española - RAE.

Esta búsqueda se realizó ajustando por término o por concepto.

3. Identificación de términos en lenguaje controlado (MeSH y DeCS):

Considerando que son dos de los tesauros más usados por sus bases de datos.

MeSH (Medical Subject Headings): Es el listado de terminología jerárquicamente organizada para la indexación y catalogación de información biomédica, como MEDLINE a través de PubMed. Este tesauro es desarrollado por la Librería Nacional de Medicina- NLM(12). DeCS (Descriptores en Ciencias de Salud): ofrece un lenguaje único en la indización de artículos de documentos científicos y académicos. También permite la recuperación de información disponible en la Biblioteca Virtual en Salud (BVS) como son LILACS, MEDLINE, $B D E N F$, BINACIS y otras. Este Tesauro fue creado por BIREME y su base ha sido desarrollada a partir de los $M e S H^{(10)}$.

4. Construcción de ecuación de búsqueda utilizando conectores boleanos:

Se construye a partir de los MeSH y DeCS con conector boleano AND. La ecuación se registra según aparecen los términos y se ingresan uno a uno en el constructor.

5. Búsqueda y recuperación de artículos en base de datos MEDLINE a través de PubMed y en La Biblioteca Virtual en Salud BVS incluyendo las bases de datos que generen resultados de búsqueda.

6. Aplicación de filtros según criterios de inclusión o necesidades del investigador. Puede ser por periodo de tiempo, bases de datos, disponibilidad, idioma, entre otros.

7. Selección de artículos según pertinencia.

8. Con los artículos seleccionados se realiza la lectura crítica para responder la pregunta formulada.

En la Figura 1 se muestra la ruta de la estrategia.

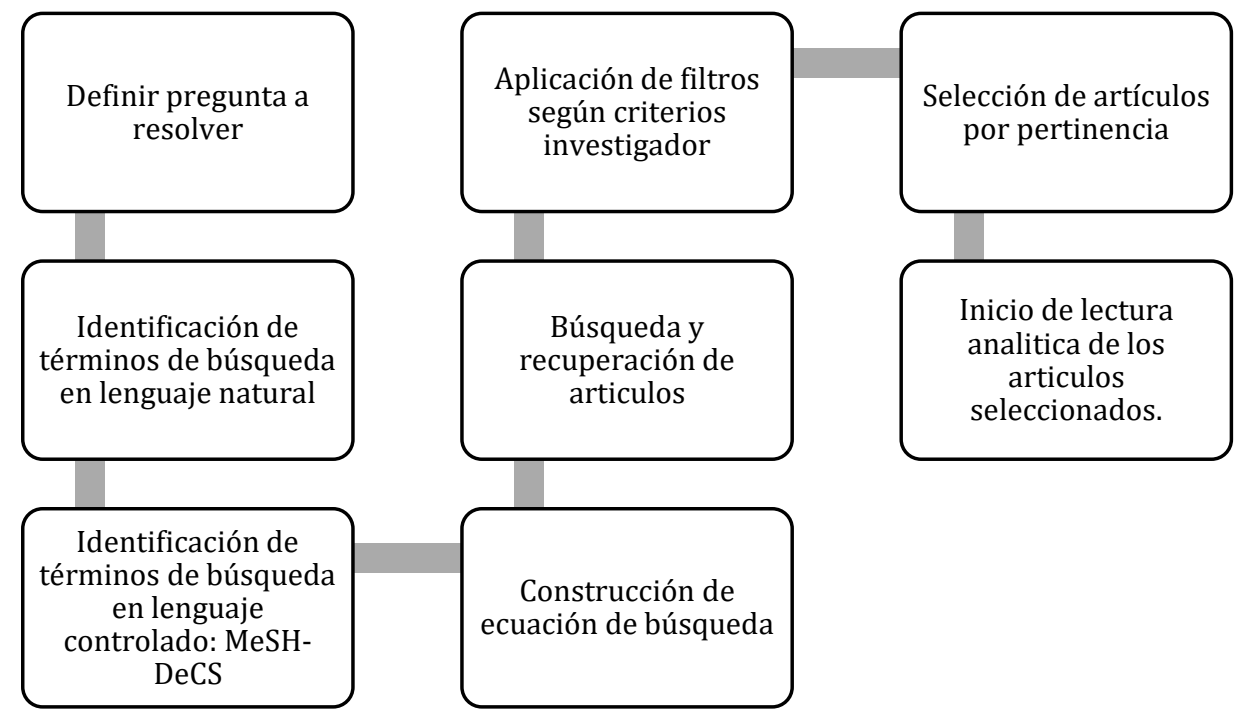

Figura 1. Ruta de búsqueda de información científica.

\section{Resultados}

Cuando una persona con discapacidad generada durante su periodo laboral debe reintegrarse a su trabajo, es necesario realizar una seria de intervenciones por parte del empleador, desde el Sistema General de Riesgos Laborales y el entorno 
social de manera que se desarrolle una intervención profesional y actualizada a partir de la evidencia científica.

\section{Definición de la pregunta a resolver}

La estrategia de búsqueda para la pregunta planteada: ¿Qué se ha investigado en el tema de reintegro laboral en las personas con discapacidad en los últimos 5 años? Generará un insumo para abordar las intervenciones desde la evidencia científica. Esta pregunta se usará para realizar la estrategia de búsqueda tanto con los $\mathrm{MeSH}$ como los DeCS.

\section{Identificación de términos de búsqueda en lenguaje natural}

Considerando la pregunta del ejemplo, se identifica dos categorías claves para realizar la búsqueda:

Los términos de búsqueda: Reintegro laboral y discapacidad.

En un primer momento se identifican los sinónimos usando diccionario de la Real Academia Española $\operatorname{RAE}^{(13) .}$

\section{Sinónimos de reintegro laboral}

Usando el diccionario el término no se registra, por lo tanto la búsqueda se realiza por palabras:

Reintegro: reintegrar

Laboral: trabajo

En lenguaje natural: reincorporación al trabajo, volver al trabajo, retornar al trabajo.

\section{Sinónimos de discapacidad}

Discapacidad: discapacitado.

En lenguaje natural: minusválido, persona con discapacidad, persona en situación de discapacidad.

En lenguaje natural: discapacidad generada por el trabajo, discapacidad a consecuencia del trabajo.

Luego, se tomaron los conceptos de reintegro laboral y discapacidad, teniendo como resultado que el reintegro laboral es definido como: "el Conjunto de acciones, actividades y procedimientos, en la que se concentra la estrategia de retorno a una actividad productiva en la misma empresa, en condiciones de desempeño eficiente, seguridad y confort"(14). La discapacidad es considerada como el: "Término genérico que incluye limitaciones en la realización de una actividad" (15) desde el contexto de trabajo, se puede complementar con el concepto de pérdida de capacidad laboral: "Pérdida de habilidades, destrezas, aptitudes y/o potencialidades de orden físico, mental y social, que permiten desempeñarse en un trabajo"(15).

\section{Identificación de términos de búsqueda en lenguaje controlado: $\mathrm{MeSH}-\mathrm{DeCS}$}

La descripción de términos establecida en el paso número dos (2) permitió identificar los términos en lenguaje controlado. Se realizó búsqueda en los Thesauros MeSH Y DeCS.

Se realizó primero búsqueda con los $M e S H$ y luego con los DeCS.

Estrategia de identificación de términos $\mathrm{MeSH}$ en PubMed: para este paso se realizó traducción al idioma inglés de todos los términos seleccionados, como se presenta a continuación:

Ejemplo de identificación de términos en idioma inglés según sinónimos de término reintegro laboral y discapacidad

Término en español y sinónimos:

- Trabajo: work, job, labor, assignment, occupation, toil, employ, enterprise.

- Empleo: job, employment, occupation, work, employ

- $\quad$ Discapacidad: disability, impairment.

- Minusválido/discapacitado: handicapped, disabled, invalid.

- $\quad$ Reintegro laboral: labor reinstatement.

- Reincorporación laboral: labor reincorporation.

- Volver al trabajo: back to work, return to work.

- Persona en situación de discapacidad: person in a situation of disability.

Una vez se establecieron los términos en inglés, se identificaron en el Tesauros los descriptores que son pertinentes a la búsqueda a realizar.

WORK: se encontraron 29 descriptores con el término WORK, de los cuales, según la definición, aplicaron para el estudio:

- Work: productive or purposeful activities. Year introduced: 1966 
- Return to work: resumption of normal work routine following a hiatus or period of absence due to injury, disability, or other reasons. Year introduced: 2013

JOB: se encontraron 17 descriptores, de los cuales, según la definición, aplicaron para el estudio:

- Employement: The state of being engaged in an activity or service for wages or salary. Year introduced: 1970

LABOR: se encontraron 19 descriptores, de los cuales 2 descriptores estaban relacionados:

- Work y Employement. Términos que ya se encuentran definidos con anterioridad.

ASSIGMENT: este término no se encontró en los descriptores

OCCUPATIONS: se encontraron 3 descriptores, los cuales en su definición no fueron pertinentes para la búsqueda.

TOIL: arroja un descriptor:

- Work: este término se describe como WORK y $L A B O R$

EMPLOY: no produjo resultado

ENTRERPRISE: presentó 20 descriptores de los cuales, según la definición, no fueron pertinentes para este estudio.

EMPLOYMENT: se encontraron 4 descriptores de los cuales uno se relacionó con la búsqueda:

- Employement: se define en el descriptor $J O B$

Siguiendo la estrategia, se realizó el mismo ejercicio con los términos relacionados con discapacidad.

DISABILITY: se encontraron 164 descriptores, los cuales, al ser revisados, 2 se relacionaron con el tema. De los otros descriptores, la mayoría están definidos según discapacidades congénitas.

- Disabled Persons: Persons with physical or mental disabilities that affect or limit their activities of daily living and that may require special accommodations. Year introduced: 1998(1963).

- Return to work: se define en el descriptor WORK.
HANDICAPPED: se encontraron cinco descriptores, de los cuales, uno según su definición aplicó para la búsqueda:

- Disabled Persons: se define en el descriptor DISABILITY.

INVALID: no arrojó resultados.

DISABLED: se encontraron 24 descriptores, de los cuales 1 según definición aplicó:

- Disabled Persons: se define en el descriptor DISABILITY y HANDICAPPED.

Un tercer paso fue realizar la búsqueda con términos combinados:

\section{LABOR REINSTATEMENT $\mathbf{y}$ LABOR} REINCORPORATION: arrojaron los mismos resultados. Se encontraron 19 descriptores de los cuales

- Work: se define en el descriptor WORK y LABOR

- $\quad$ Employment: se define en el descriptor JOB

BACK TO WORK: arrojó un descriptor

- $\quad$ Return to work: descrito en los Tesauros WORK y DISABILITIES

RETURN TO WORK: arrojó un descriptor:

- Return to work: descrito en los tesauros WORK, DISABILITIES y BACK TO WORK

DISABILITY GENERATED BY WORK: no arrojó resultados.

DISABILITY DUE TO WORK: arrojó un resultado:

- $\quad$ Return to Work: descrito en los tesauros WORK, DISABILITIES, BACK TO WORK y RETURN TO WORK.

PERSON IN A SITUATION OF DISABILITY: no arrojó resultados.

\section{Construcción de ecuación de búsqueda usando MeSH(12)}

Una vez se realizó la búsqueda en el Tesauros, considerando la pregunta ejemplo se planteó dos ecuaciones de búsqueda que unieron los dos descriptores que más representaron el tema elegido, siendo así, se estableció los descriptores: Return to work (tema) y Disabled persons (población). 
A continuación, se presentan las dos ecuaciones de búsqueda: una en general y la otra usando el Major topic.

a. Ecuación de búsqueda general según los descriptores pertinentes y seleccionados acorde con la definición de reintegro laboral:

("return to work"[MeSH Terms] OR ("return"[All Fields] AND "work"[All Fields]) OR "return to work"[All Fields]) AND ("disabled persons"[MeSH Terms] OR ("disabled"[All Fields] AND "persons"[All Fields]) OR "disabled persons"[All Fields])

b. Ecuación de búsqueda usando Major Topic:

"Return to Work"[Majr] AND "Disabled Persons"[Majr]

\section{Búsqueda y recuperación de artículos}

Una vez se estableció la ecuación de búsqueda se procedió a realizar la búsqueda en Medline a través de PubMed.

La primera búsqueda se realizó de manera independiente con cada descriptor y luego con la combinación de los dos.

Al realizar búsqueda con cada descriptor se obtuvo:

Return to work: 14.778 artículos.

Disabled persons: 63.421 artículos.

La siguiente búsqueda se realizó usando el conector boleano "AND". Así mismo, luego, para obtener mayor especificidad en la búsqueda se usa el Major Topic, lo que permite que se incluyan artículos que más se relacionan con el concepto.

En la tabla 1 se identifica el número de artículos recuperados comparando según la ecuación planteada.

Tabla 1. Resultado comparativo de artículos recuperados según ecuación de búsqueda MeSH

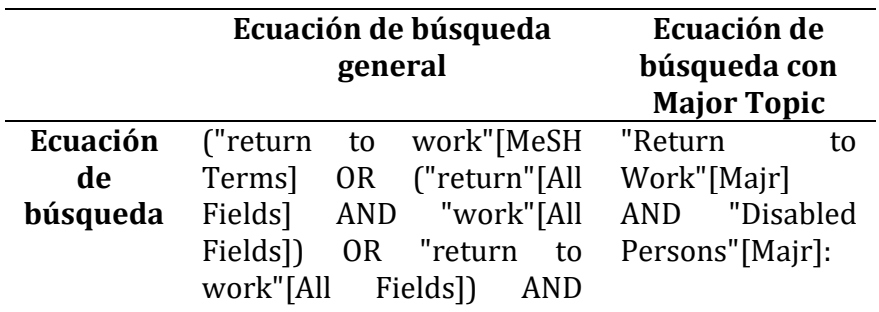

("disabled persons"[MeSH

Terms] OR ("disabled"[All

Fields] AND "persons"[All

Fields]) OR "disabled

persons"[All Fields])

Total

581 artículos

87 artículos

sin filtro

Se identifica que al realizar la búsqueda usando Major Topic los resultados son más centrados al concepto y esto permite identificar los artículos más pertinentes según los descriptores y su definición.

En la segunda búsqueda con la ecuación de búsqueda "Return to Work"[Majr] AND "Disabled Persons"[Majr] se obtuvieron 11 artículos que están incluidos en los resultados de la primera búsqueda.

\section{Aplicación de filtros según criterios investigador}

Considerando la pregunta ejemplo planteada se propusieron dos filtros en la búsqueda:

a. Libre acceso: permite acceder de manera gratuita al artículo completo y así poder realizar la lectura y análisis

b. Periodo: últimos 5 años.

A continuación, se presenta la figura 2 de la estrategia de búsqueda usando MeSH en Medline a través de PubMed y cantidad de artículos

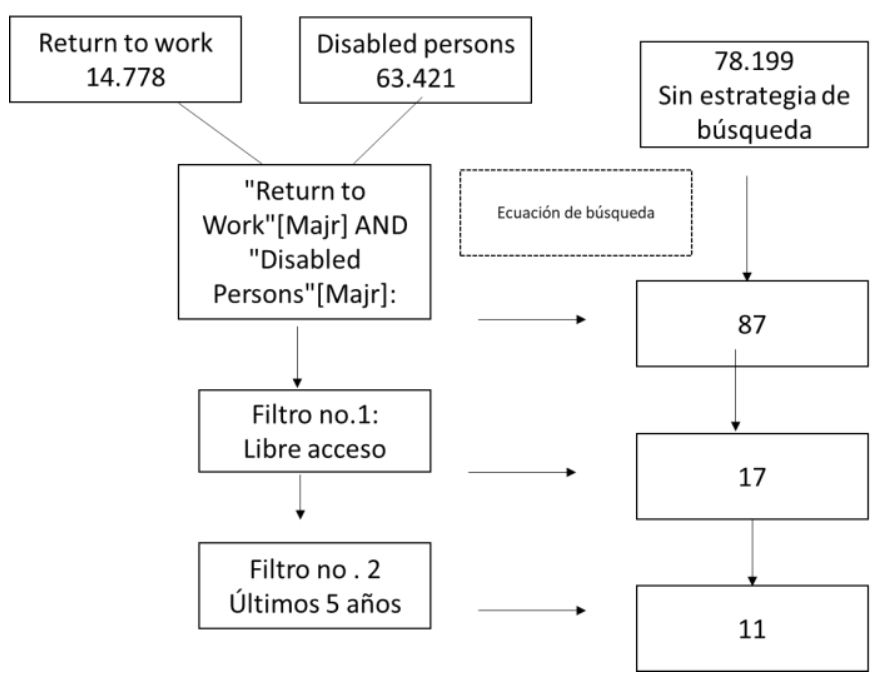

Figura 2. Estrategia de búsqueda y cantidad de artículos recuperados en cada etapa.

recuperados en cada etapa.

\section{Selección de artículos por pertinencia}


Posterior a esto, tomando los resultados de la ecuación de búsqueda "Return to Work"[Majr] AND "Disabled Persons"[Majr], se realizó comprobación de la pertinencia de los artículos siguiendo el esquema:

a. Por título: 9 artículos pertinentes en primera instancia

b. Por resumen: 11 (total) artículos pertinentes.

El total de los artículos recuperados siguiendo la estrategia de búsqueda son pertinentes y se ajustan a los criterios para dar respuesta a la pregunta planteada.

Se presenta el listado de los artículos recuperados:

1. Assessing factors associated with long-term work disability after cancer in Belgium: a populationbased cohort study using competing risks analysis with a 7-year follow-up(16).

2. Labour market participation and sick leave among patients diagnosed with myasthenia gravis in Denmark 1997-2011: a Danish nationwide cohort study(17).

3. Work disabilities and unmet needs for health care and rehabilitation among jobseekers: a community-level investigation using multidimensional work ability assessments(18).

4. The impact on health of employment and welfare transitions for those receiving out-of-work disability benefits in the UK(19).

5. Effects of graded return-to-work: a propensityscore-matched analysis(20).

6. Socially constructed 'value' and vocational experiences following neurological injury (21).

7. Prognostic factors for disability and sick leave in patients with subacute non-malignant pain: a systematic review of cohort studies (22).

8. Subjective perceptions as prognostic factors of time to fitness for work during a 4-year period after inpatient rehabilitation for orthopaedic trauma(23).

9. Return-to-Work Within a Complex and Dynamic Organizational Work Disability System ${ }^{(24)}$.

10. Predictors of permanent work disability among $\leq 50$-year-old patients undergoing percutaneous coronary intervention(25).

11. Is temporary employment a risk factor for work disability due to depressive disorders and delayed return to work? The Finnish Public Sector Study(26)
A continuación, se presenta los resultados de cada uno de los pasos de la estrategia de búsqueda utilizando los DeCS. Para este punto, los pasos 1 y 2 son los mismos usados para los $M e S H$, una vez se cumplen los pasos 1 y 2 , se continua con el paso no. 3.

\section{Identificación de términos de búsqueda en lenguaje controlado: DeCS}

Para este paso se tomaron los términos en español y se realizó la normalización según los $D e C s$.

TRABAJ0: se encontraron 61 descriptores.

- Empleo: la condición de ser contratado en una actividad o servicio por un salario o pago. Fuerza de trabajo: Potencial humano, de una determinada población, económicamente activa.

- Trabajo: actividades productivas o con un determinado propósito.

- Reinserción al trabajo: reanudación de la rutina de trabajo normal después de un hiato o período de ausencia debido a una lesión, discapacidad, u otra razón.

EMPLEO: se encontraron 4 descriptores.

- Empleo: descripción en Trabajo.

- Trabajadores: personas que se ocupan en algún oficio, profesión o actividad remunerada o no, con o sin vínculo laboral formal.

DISCAPACIDAD: se encontraron 18 descriptores.

- Personas con discapacidad: personas con discapacidades físicas o mentales que afectan o limitan sus actividades de la vida diaria y que pueden requerir adaptaciones especiales.

Los siguientes términos no arrojaron resultados: minusválido, discapacitado, reintegro laboral, reincorporación laboral, volver al trabajo, discapacidad generada por el trabajo, discapacidad consecuencia del trabajo, persona en situación de discapacidad.

Considerando la definición de reintegro laboral se tomaron los descriptores: reinserción al trabajo y persona con discapacidad.

\section{Construcción de ecuación de búsqueda}

Considerando la pregunta ejemplo se planteó dos ecuaciones de búsqueda que unieron los dos descriptores que más representaron el tema elegido: 
REINSERCIÓN AL TRABAJO: (tw:(reinsercion al trabajo)), se recuperaron 1.972 artículos, 57 de ellos en LILACS y 1813 en MEDLINE y PERSONA CON DISCAPACIDAD: (tw:(persona con discapacidad)), Se recuperaron 19.107 artículos, 55.698 están en MEDLINE y 2.287 en LILACS.

\section{Búsqueda y recuperación de artículos}

Una vez se estableció la ecuación de búsqueda se procedió a realizarla en el portal Regional de la BVS, se tomaron los descriptores anteriores y se unieron usando el conector boleano "AND". La primera búsqueda se llevó a cabo de manera independiente con cada descriptor y luego, la combinación de los dos obteniendo 157 artículos con la ecuación: (tw:(reinserción al trabajo)) AND (tw:(persona con discapacidad))

\section{Aplicación de filtros según criterios investigador}

Considerando la pregunta ejemplo planteada se propuso dos filtros en la búsqueda:

a. Libre acceso: permite acceder de manera gratuita al artículo completo y así poder realizar la lectura y análisis. Este filtro se aplica al considerar los profesionales que no tienen suscripción a bases de datos, ni revistas indexadas. Permite la recuperación inmediata de la información que se requiere.

b. Periodo de tiempo: últimos 5 años.

Los resultados que arrojó la ecuación de búsqueda fueron: según el primer filtro: base de datos diferente a MEDLINE en un periodo de tiempo de los últimos 5 años: en IBECS: 5 artículos, en BDENF: 1 artículo y en LILACS: 1 artículo para un total de 7 artículos.

Para el segundo filtro, artículos de libre acceso, se

\begin{tabular}{|c|c|c|}
\hline $\begin{array}{c}\text { Reinserción al } \\
\text { trabajo } \\
1.972\end{array}$ & $\begin{array}{c}\text { Persona con } \\
\text { discapacidad } \\
55.698\end{array}$ & $\begin{array}{c}57.670 \\
\text { Sin estrategia de } \\
\text { búsqueda }\end{array}$ \\
\hline
\end{tabular}

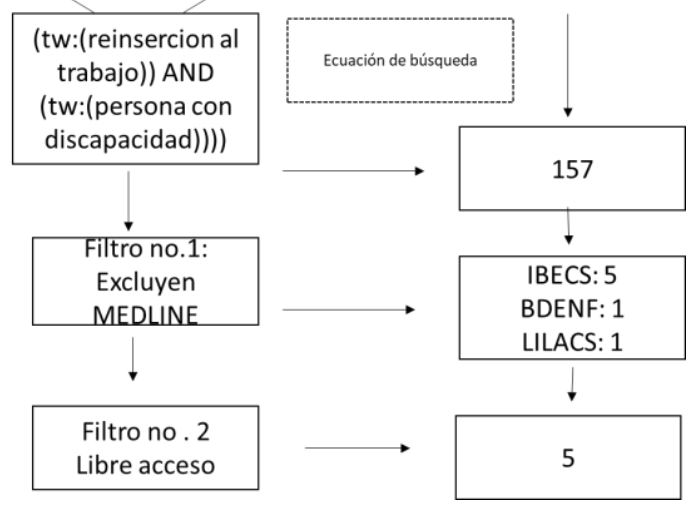

Figura 3. Esquema de estrategia de búsqueda con DeCS a través de BVS y número de artículos recuperados en cada etapa. obtuvo un total de 5 artículos

En la figura 3 se presenta el esquema de la estrategia y los resultados después de aplicar los filtros.

\section{Selección de artículos por pertinencia}

Posterior a esto, tomando los resultados de la ecuación de búsqueda "(tw:(reinserción al trabajo)) AND (tw:(persona con discapacidad))", se realizó comprobación de la pertinencia de los artículos siguiendo el esquema por título y por resumen.

Los artículos recuperados según la ecuación de búsqueda son:

1. No apto pero no incapacitado. La controversia del ser o no ser / Not fit but not disabled. To be or not to be ${ }^{(27)}$.

2. Corticoides transforaminales en la radiculopatía lumbosacra: valoración del dolor y la discapacidad(28).

3. An empowerment model of entrepreneurship for people with disabilities in the United States(29).

4. Qualidade de vida em pacientes com lesão medular / Calidad de vida en pacientes con lesión medular / Quality of life in patients with spinal cord injury(30).

Luego, se realizó comprobación de la pertinencia de los artículos siguiendo el esquema:

El artículo: Quelidade de vida em pacientes com lesao medular: se encuentra en LILACS y BDENFE, por lo tanto, son cuatro los artículos recuperados.

a. Por título: tres artículos pertinentes en primera instancia

b. Por resumen: tres (total) de artículos pertinentes.

El total de los artículos recuperados siguiendo la estrategia de búsqueda son pertinentes y se ajustan a los criterios para cumplir con el objetivo planteado.

\section{Lectura analítica de los artículos seleccionados}

Este paso aplica para la búsqueda realizada tanto con los descriptores MeSH: once artículos y DeCS con cuatro artículos.

Se considera la pregunta ejemplo: ¿Qué se ha investigado en el tema de reintegro laboral en las personas con discapacidad en los últimos 5 años? 
Con los artículos recuperados según la estrategia de búsqueda se realiza lectura analítica desde la hermenéutica, se genera una matriz de análisis y finalmente se agrupan los resultados acordes con los referentes de discapacidad y reintegro laboral. Según los artículos seleccionados, producto de la estrategia de búsqueda en reintegro laboral de personas con discapacidad se ha investigado en temas según: población, diagnóstico médico, capacidad valorada para trabajar, modelos y enfoques para abordar el reintegro laboral en las personas con discapacidad; factores predictores del reintegro laboral y el reintegro temprano.

a. Población según diagnóstico médico: personas o datos de personas con diagnósticos de cáncer(16), miastenia grave(17), discapacidad laboral(18), lesión neurológica adquirida(28,30), trauma ortopédico(20), dolor(22), alteraciones $\operatorname{cardiacas}^{(20,25)}$, depresión(26).

b. Capacidad valorada: Categorías para trabajar. Después de una valoración multidisciplinar, el médico conceptúa unas categorías que proyectan al trabajador en el medio laboral: 1. Buena habilidad para trabajar, 2. Buena habilidad para trabajar después de RTW, 3. Con capacidad para un trabajo temporal o de preparación y 4. No puede trabajar(18). En algunas oportunidades se propone el emprendimiento como opción de reintegro laboral(29).

c. Modelos y enfoques para abordar el reintegro laboral en personas con discapacidad:

- Desde el enfoque de un posible modelo interdisciplinar: Se identifica la necesidad de realizar abordaje interdisciplinar que incluye al trabajador, la empresa, el equipo de rehabilitación.

- Desde un enfoque social y cultural: La concepción de poco valor laboral otorgado a las personas con discapacidad que afecta el proceso de reintegro laboral, en ocasiones se identifica discriminación en el ámbito laboral(27, 29).

- Desde la organización: el papel del empleador en facilitar el reintegro laboral con alternativas como gimnasia laboral, reintegro temprano, estabilidad laboral, aceptación de la condición de salud del trabajador.

d. Factores predictores de reintegro laboral de las personas con discapacidad. Los artículos mencionan que se debe considerar al momento del reintegro laboral:

- Tiempo de incapacidad: entre menos tiempo de incapacidad mayores oportunidades de reintegro laboral.

- Género: Las mujeres tienen más oportunidad de reintegrarse al trabajo.

- Edad: A menor edad del trabajador, más oportunidad de reintegrarse al trabajo.

- Cargo: Los trabajadores con cargos administrativos.

- Tipo de diagnóstico: Generalmente asociado con el tiempo de incapacidad y percepción que tiene el trabajador sobre sus capacidades.

- Modelo de abordaje: Modelo multidisciplinar con diferentes profesionales.

- Tipo de vinculación laboral: Cuando se tiene un contrato temporal el reintegro laboral tiene menos opciones.

e. Reintegro temprano: Considerado como un protector de la salud que disminuye la posibilidad de generar discapacidad, aportar en la economía social, reducir impacto por pensión por discapacidad(19-20).

\section{Discusión}

Sobre el reintegro laboral existe diversidad de abordajes, métodos, intereses que hacen sea complejo al momento de planearlo con personas con discapacidad. Al realizar búsqueda de información avalada por medios académicos, que orienten en el abordaje del tema, se encuentra que sólo con el término de reintegro laboral se recuperan en Medline a través de PubMed 14.778 artículos. Lo anterior llevaría a pensar que sobre el tema se está investigando mucho $y$ que se podrá tomar información relevante que aporte en el quehacer profesional en el tema específico. Sin embargo, además de ser mucha información para analizar, no toda es específica para la población con discapacidad y tampoco pertinente para los intereses de búsqueda. Con la estrategia de búsqueda(11) propuesta se evidencia que se puede obtener resultados que responden a una pregunta planteada $^{(8)}$ y ser efectivos en la recuperación de información al seguir cada uno de los pasos, partiendo del planteamiento de la pregunta. Los resultados obedecen a la aplicación de filtros que si bien, limitan el número de artículos recuperados, 
permite acceder a la información específica que se pretende encontrar en un momento dado.

La pregunta para responder en ocasiones no es considerada como parte de la búsqueda y esto lleva a obtener resultados descontextualizados que no se relacionen con el interés particular. Según se plantee la pregunta se identifican los términos clave que permiten establecer el lenguaje natural ${ }^{(9)}$ y a partir de este el lenguaje controlado(10), lo cual facilita la recuperación de información con términos estandarizados $\mathrm{y}$ por lo tanto, controlados académicamente. Se identificó con esta investigación que existen palabras que al ser traducidas al idioma inglés tienen un significado - connotación diferente que afecta los resultados de búsqueda. Para establecer los descriptores es necesario tener claridad sobre el o los conceptos que se abordaran.

Un ejemplo de lo anterior y que cobra más relevancia para evidenciar la efectividad de una estrategia de búsqueda, es el término natural de reintegro laboral, el cual al normalizarlo según los DeCS no registra como tal y por lo tanto se requiere usar otro término para lograr recuperar información, lo que en la estrategia de búsqueda es planteado como identificación de términos de búsqueda en lenguaje controlado MsSH - DeCS (figura 1). Por esto, se considera que la experticia en el tema es un factor que facilita la búsqueda debido al conocimiento y por lo tanto, la aplicación para identificar los Tesauros pertinentes.

Considerando la pregunta de ejemplo “ ¿Qué se ha investigado en el tema de reintegro laboral en las personas con discapacidad en los últimos 5 años? y los artículos recuperados según la estrategia de búsqueda, se logra evidenciar los asuntos que se están investigando en el tema de reintegro laboral de las personas con discapacidad y que fueron consolidados por categorías: población según diagnóstico médico, capacidad valorada por categorías para trabajar, modelos y enfoques para abordar el reintegro laboral en personas con discapacidad, factores predictores de reintegro laboral de las personas con discapacidad y reintegro temprano.

Un aspecto relevante, es la mirada del trabajo en las personas con discapacidad como un predictor de la condición de salud y vulnerabilidad(21,27), lo cual en la actualidad, está reflejando barreras para la participación de las personas con discapacidad en el mercado laboral, esto, acorde con lo planteado por la OMS $^{1}$, que manifiesta el riesgo de menor participación en actividades de la vida diaria siendo una de ellas el trabajo.

Así mismo se plantea unas categorías relacionadas con la capacidad para trabajar: 1 . Buena habilidad para trabajar 2. Buena habilidad para trabajar después del reintegro laboral, 3. Con capacidad para un trabajo temporal o de preparación al trabajo y 4 . No puede trabajar, que serán usadas como directrices para el reintegro laboral.

En Colombia también se tiene la orientación tanto para calificar la pérdida de capacidad laboral y las modalidades de reintegro(2): sin modificaciones, con modificaciones, reubicación temporal, reubicación y orientación vocacional. Estas calificaciones, si bien dan una guía para abordar el reintegro de la persona con discapacidad, requieren ser analizadas en detalle, considerando el enfoque multidisciplinar(3) con una mirada del trabajador como ser humano y por lo tanto, integral $\mathrm{y}$ en un dinamismo constante(19).

Por el mismo dinamismo del ser humano, la forma en que se aborda el reintegro laboral amerita un trabajo individualizado según la condición de salud, situaciones y experiencias de vida; esto se refleja en los artículos rescatados con la ecuación de búsqueda, identificando que según el diagnóstico se tendrá tiempo de incapacidad diferente, recuperación y tipo de rehabilitación diferente(25), así como las mismas expectativas y autoconcepto del trabajador(22).

Si bien se espera que el abordaje del reintegro laboral sea interdisciplinar ${ }^{3}$, los artículos rescatados evidencian acciones desarticuladas con abordajes desde diferentes miradas(27) lo cual disminuye la participación y empoderamiento tanto del trabajador como del sistema de salud y de riesgos laborales y por lo tanto, afecta el proceso de reintegro laboral ya sea con un reintegro tardío o no reintegro laboral.

En la misma línea de lo planteado por la OMS(1) la menor participación en el mercado laboral de las personas con discapacidad, puede ser producto de un concepto sociocultural y que se evidencia en la discriminación(27), conceptos relacionados con poca capacidad y desconocimiento del impacto de las condiciones de salud en el desempeño laboral y actividades de la vida diaria en general(21). 


\section{Conclusiones}

Con este estudio se logró evidenciar la pertinencia de una estrategia búsqueda para recuperar publicaciones científicas, para este caso, reintegro laboral de las personas con discapacidad obteniendo resultados agrupados por categorías: población según diagnóstico médico, capacidad valorada y categorías para trabajar, modelos y enfoques para abordar el reintegro laboral en personas con discapacidad, factores predictores de reintegro laboral de las personas con discapacidad y reintegro temprano, que dan orientación a los profesionales para realizar sus propuestas de intervención en el tema acorde con los intereses y necesidades.

La identificación de términos de búsqueda en lenguaje controlado, evidencia una necesidad de profundizar en la conceptualización de los términos usados, de esa manera, tener un conceso no sólo académico, sino social del tema de reintegro laboral.

\section{Recomendaciones}

Desde los procesos en el actuar profesional, se recomienda fortalecer en los profesionales de salud, las competencias en búsqueda y recuperación de información científica.

La estrategia de búsqueda de información científica presentada en este artículo, permite cambiar los descriptores según sea el interés del profesional, por lo tanto, es recomendada para diferentes temas que requieran profundizar, conocer, aclarar.

Se recomienda a los profesionales en salud realizar búsquedas de información en bases de datos a través de la estrategia planteada, para así obtener datos académicos y científicos avalados por la comunidad experta en el tema.

A partir del uso de la estrategia de búsqueda, específicamente en el paso 3: Para la recuperación de artículos usando los DeCS, siendo el principal idioma el español, se hace evidente las pocas publicaciones en el tema por lo tanto, es un llamado de atención a los investigadores y académicos a investigar y publicar para fortalecer así mismo la práctica profesional.

Con este artículo se evidencia la necesidad de ampliar el libre acceso a los artículos de investigación y promoverlo como una política entre los investigadores.
Conflicto de intereses: no se presenta conflicto de interés.

\section{Referencias}

1. Organización Mundial de la Salud. Discapacidades [Internet]. 2018. Available from: http://www.who.int/topics/disabilities/es/

2. Ministerio de la Protección Social Dirección General de Riesgos Profesionales. Manual de procedimientos para la rehabilitación y reincorporación ocupacional de los trabajadores en el sistema general de riesgos profesionale [Internet]. 2010 [cited 2018 Jun 1]. Available from: http://fondoriesgoslaborales.gov.co/documents/Publicacio nes/Manuales/MANUAL-PARA-REHABILITACION-2012.pdf

3. Cuervo Díaz DE. Prevención y Manejo de la Discapacidad para Trabajar: Un Análisis al Sistema de Riesgos Laborales Colombiano [Internet]. 2016 [cited 2019 Mar 15]. Available from: https://dianacuervophd.com/wpcontent/uploads/2018/09/Tesis.pdf

4. Google [Internet]. California: Google; 1998 [updated 2020; cited 2010 Mar]. Available from: https://www.google.es/

5. Google Scholar. Reintegro laboral de personas con discapacidad [Internet]. 2019 [cited 2019 Mar 14]. Available from:

https://scholar.google.es/scholar?hl=es\&as_sdt=0\%2C5\&q =reintegro+laboral+de+personas+con+discapacidad \&btnG=

6. NCBI. PubMed - NCBI [Internet]. 2019 [cited 2019 Mar 15]. Available from: https://www.ncbi.nlm.nih.gov/pubmed

7. Ávila de Tomás J, Portillo Boyero B, Pajares Izquierdo J. Calidad en la información biomédica existente en Internet [Internet]. Vol. 28, Atención primaria. 2001 [cited 2019 Mar 11]. Available from: http://www.accesible.org/wmc/wmcborrador1.htm.

8. Mamédio C, Santos C, Andrucioli De Mattos Pimenta C, Roberto M, Nobre C. ESTRATEGIA PICO PARA LA CONSTRUCCIÓN DE LA PREGUNTA DE INVESTIGACIÓN Y LA BÚSQUEDA DE EVIDENCIAS A ESTRATÉGIA PICO PARA A CONSTRUÇÃO DA PERGUNTA DE PESQUISA E BUSCA DE EVIDÊNCIAS. Rev latino-am ENfermagen [Internet]. 2007 [cited 2019 Jan 29];15(3). Available from: www.eerp.usp.br/rlaeArtigodeAtualização

9. Peña.C. Organización del Conocimiento: Bibliotecología y términología. In: México UNA de, editor. México; 2009 [cited 2019 Feb 12]. Available from: https://s3.amazonaws.com/academia.edu.documents/3173 2425/organizacion_del_conocimiento.pdf?AWSAccessKeyId =AKIAIWOWYYGZ2Y53UL3A\&Expires=1549986923\&Signa ture $=c 3$ FiqzG8Uc1MT9zIWCr7cI6rCmA\%3D\&responsecontent-disposition=inline $\% 3 \mathrm{~B}$ filename\%3Dorganizacion_del

10. BIREME / OPS / OMS. Descriptores en Ciencias de la Salud: DeCS [Internet]. ed.. Sao Paulo (SP); 2017 [cited 2019 Feb 12]. Available from: http//decs.bvsalud.org/E/homepagee.htm

11. Gómez-Luna E, Fernando-Navas D, Aponte-Mayor G, Betancourt-Buitrago LA. Metodología para la revisión bibliográfica y la gestión de información de temas científicos, a través de su estructuración y sistematización TT - Literature review methodology for scientific and information management, through its structuring and systema. Dyna. 2014;81(184):158-63. 
12. NIH. Medical Subject Headings - MeSH [Internet]. U.S. National Library of Medicine; [cited 2019 Feb 12]. Available from: https://www.nlm.nih.gov/MeSH/MeSHhome.html

13. Real Academia Española. Real Academia Española [Internet]. 2019 [cited 2019 Mar 12]. Available from: http://www.rae.es/

14. Ministerio de la Protección Social. Instrumentación del Manual Guía de Procedimientos de Rehabilitación y Reincorporación Ocupacional de los Trabajadores en el Sistema General de Riesgos Profesionales [Internet]. [cited 2018 Nov 28]. Available from: http://acripbolivar.org/descargas/paginas.pdf

15. Ministerio del Trabajo. Manual Único para la Calificación de la Pérdida de la Capacidad Laboral y Ocupacional- Decreto 1507 de 2014. Ministerio del Trabajo. Bogotá, Colombia. [Internet]. Disponible en: https://www.mintrabajo.gov.co/documents/20147/51963 /Manual+Unico+de+Calificaciones+Decreto.pdf/7d224908ef78-1b90-0255-f62a3e409e4c

16. Kiasuwa M, Nicolale A, Goetghebeur E, et al. Assessing factors associated with long-term work disability after cancer in Belgium: a population-based cohort study using competing risks analysis with a 7-year follow-up. BMJ Open [Internet]. 2018 [cited 2019 Feb 12];8:14094. Available from: http://bmjopen.bmj.com/

17. Frost A, Svendsen ML, Rahbek J, Stapelfeldt CM, Nielsen CV, Lund T. Labour market participation and sick leave among patients diagnosed with myasthenia gravis in Denmark 1997-2011: a Danish nationwide cohort study. BMC Neurol [Internet]. 2016 Nov 17 [cited 2019 Mar 12];16(1):224. Available http://www.ncbi.nlm.nih.gov/pubmed/27855651

18. Kerätär R, Taanila A, Jokelainen J, Soukainen J, Ala-Mursula L. Work disabilities and unmet needs for health care and rehabilitation among jobseekers: a community-level investigation using multidimensional work ability assessments. Scand J Prim Health Care [Internet]. 2016 [cited 2019 Feb 12];34(4):343-51. Available from: https://www.tandfonline.com/action/journalInformation?j ournalCode=ipri20

19. Curnock E, Leyland AH, Popham F. The impact on health of employment and welfare transitions for those receiving outof-work disability benefits in the UK. Soc Sci Med. 2016;162:1 - 10. doi:10.1016/j.socscimed.2016.05.042

20. Bethge M. Effects of graded return-to-work: a propensityscore-matched analysis. Scand J Work Environ Health [Internet]. 2016 [cited 2019 Feb 12];42(4):273-9. Available from: http://www.ncbi.nlm.nih.gov/pubmed/27074050

21. Fadyl JK, Payne D. Socially constructed "value" and vocational experiences following neurological injury. Disabil Rehabil [Internet]. 2016 [cited 2019 Feb 12];38(22):2165$77 . \quad$ Available from: http://dx.doi.org/10.3109/09638288.2015.1116620

22. Valentin GH, Pilegaard MS, Vaegter HB, Rosendal M, Ørtenblad L, Vaeggemose U, et al. Prognostic factors for disability and sick leave in patients with subacute nonmalignant pain: a systematic review of cohort studies. BMJ Open [Internet]. 2015 [cited 2019 Feb 12]; Available from: https://bmjopen.bmj.com/content/6/1/e007616

23. Vuistiner P, Luthi F, Erhart P, Scholz S, Dériaz O. Subjective perceptions as prognostic factors of time to fitness for work during a 4-year period after inpatient rehabilitation for orthopaedic trauma. Swiss Med Wkly [Internet]. 2015 Dec
28 [cited 2019 Mar 12];145(5152). Available from: http://doi.emh.ch/smw.2015.14235

24. Jetha A, Pransky G, Fish J, Hettinger LJ. Return-to-Work Within a Complex and Dynamic Organizational Work Disability System. J Occup Rehabil [Internet]. 2016 [cited 2019 Mar 12];26(3):276-85. Available from: http://www.ncbi.nlm.nih.gov/pubmed/26547909

25. Gunn J, Kiviniemi T, Biancari F, Kajander O, Mäkikallio T, Eskola M, et al. Predictors of permanent work disability among $\leq 50$-year-old patients undergoing percutaneous coronary intervention. Scand J Work Environ Health [Internet]. 2015 Sep 1 [cited 2019 Mar 12];41(5):460-6. Available from: http://www.ncbi.nlm.nih.gov/pubmed/26079321

26. Ervasti J, Vahtera J, Virtanen P, Pentti J, Oksanen T, Ahola K, et al. Is temporary employment a risk factor for work disability due to depressive disorders and delayed return to work? The Finnish Public Sector Study. Scand J Work Environ Health [Internet]. 2014 Jul [cited 2019 Mar 12];40(4):343-52. Available from: http://www.ncbi.nlm.nih.gov/pubmed/24664298

27. Pardo J. No apto pero no incapacitado. La controversia del ser o no ser. Med Segur Trab (Madr) [Internet]. 2017 [cited 2019 Mar 12];63(247):131-58. Available from: http://scielo.isciii.es/scielo.php?script=sci_arttext\&pid=S04 65-546X2017000200131\&lng=es\&nrm=iso

28. Papa de la Rosa P. Corticoides transforaminales en la radiculopatía lumbosacra: valoración del dolor y la discapacidad. Rev la Soc Española del Dolor [Internet]. 2014 Aug [cited 2019 Mar 12];21(4):219-25. Available from: http://scielo.isciii.es/scielo.php?script=sci_arttext\&pid=S11 34-80462014000400006\&lng=en\&nrm=iso\&tlng=en

29. Balcazar FE, Kuchak J, Dimpfl S, Sariepella V, Alvarado F. An empowerment model of entrepreneurship for people with disabilities in the United States. Psychosoc Interv [Internet]. 2014 May [cited 2019 Mar 12];23(2):145-50. Available from:

http://journals.copmadrid.org/pi/article.php?id=999600eb 275cc7196161261972daa59b

30. França ISX de, Coura AS, Sousa FS de, Almeida PC de, Pagliuca LMF. Qualidade de vida em pacientes com lesão medular. Rev Gaúcha Enferm [Internet]. 2013 Mar [cited 2019 Mar 12];34(1):155-63. Available from: http://www.scielo.br/scielo.php?script=sci_arttext\&pid=S1 983-14472013000100020\&lng=pt\&tlng=pt 